\title{
Evaluation of 3D multi-contrast joint intra- and extracranial vessel wall cardiovascular magnetic resonance
}

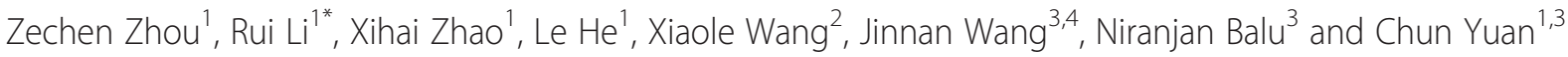

\begin{abstract}
Background: Multi-contrast vessel wall cardiovascular magnetic resonance (CMR) has demonstrated its capability for atherosclerotic plaque morphology measurement and component characterization in different vasculatures. However, limited coverage and partial volume effect with conventional two-dimensional (2D) techniques might cause lesion underestimation. The aim of this work is to evaluate the performance in a) blood suppression and b) vessel wall delineation of three-dimensional (3D) multi-contrast joint intra- and extracranial vessel wall imaging at 3T.

Methods: Three multi-contrast 3D black blood (BB) sequences with T1, T2 and heavy T1 weighting and a custom designed 36-channel neurovascular coil covering the entire intra- and extracranial vasculature have been used and investigated in this study. Two healthy subjects were recruited for sequence parameter optimization and twenty-five patients were consecutively scanned for image quality and blood suppression assessment. Qualitative image scores of vessel wall delineation as well as quantitative Signal-to-Noise Ratio (SNR) and Contrast-to-Noise Ratio (CNR) were evaluated at five typical locations ranging from common carotid arteries to middle cerebral arteries.

Results: The 3D multi-contrast images acquired within $15 \mathrm{mins}$ allowed the vessel wall visualization with $0.8 \mathrm{~mm}$ isotropic spatial resolution covering intra- and extracranial segments. Quantitative wall and lumen SNR measurements for each sequence showed effective blood suppression at all selected locations $(P<0.0001)$. Although the wall-lumen CNR varied across measured locations, each sequence provided good or adequate image quality in both intra- and extracranial segments.
\end{abstract}

Conclusions: The proposed 3D multi-contrast vessel wall technique provides isotropic resolution and time efficient solution for joint intra- and extracranial vessel wall CMR.

Keywords: Atherosclerosis, Vessel wall imaging, Carotid artery, Intracranial artery, 3D-MERGE, SNAP, VISTA, Magnetic resonance

\section{Background}

Atherosclerosis is a major cause of ischemic stroke and can develop in multiple vascular beds $[1,2]$. The presence of concurrent intra- and extracranial atherosclerosis is found to be prevalent in stroke patients [3]. In recent years, BB Vessel Wall Imaging (VWI) technique using cardiovascular magnetic resonance (CMR) has been validated to be an effective method for characterizing atherosclerotic plaque and demonstrated its potential for risk

\footnotetext{
* Correspondence: leerui@tsinghua.edu.cn

${ }^{1}$ Center for Biomedical Imaging Research, Department of Biomedical Engineering, School of Medicine, Tsinghua University, Beijing, China Full list of author information is available at the end of the article
}

stratification. Clinically, the most widely used VWI technique is $2 \mathrm{D}$ multi-contrast $\mathrm{BB}$ sequences $[4,5]$. The multi-contrast approach ensures the definition of vessel luminal surface condition, plaque distribution, and plaque burden as well as the detection of key plaque components [6-8]. But the 2D approach is limited in longitudinal coverage and partial volume effect caused by long acquisition time and large slice thickness respectively. This can be further exacerbated by the tortuosity of blood vessels particularly for intracranial arteries.

Recently, several high resolution 3D isotropic BB sequences at $3 \mathrm{~T}$ have been proposed to improve the accuracy of plaque burden measurement and plaque components 
detection for either intracranial VWI $[9,10]$ or extracranial carotid VWI [11-13] in comparison to 2D imaging. In addition, previous studies using $2 \mathrm{D}$ multi-contrast carotid CMR have identified that a basic set of sequences including T1, T2 and heavy T1 weightings can be used for plaque imaging [14-16]. Since 3D Motion Sensitized Driven Equilibrium (MSDE) prepared Rapid Gradient Echo (3D-MERGE), T2-weighted Volumetric ISotropic Turbo spin echo Acquisition (VISTA), and Simultaneous Noncontrast Angiography and intraPlaque hemorrhage (SNAP) together meet this multi-contrast requirement, they have the potential to be optimized and applied for joint intra- and extracranial screening of atherosclerosis.

However, to jointly delineate intra- and extracranial vessel wall using 3D multi-contrast sequences, several challenges need to be addressed. a) Imaging coverage and overall scan time. Simply utilizing multi-station or dual stack [17] strategy may cause misregistration between stations or stacks and lead to long scan times. b) Effective blood suppression in a large region where various blood flow pattern, direction, and velocities co-exist must be considered by specific blood suppression techniques. c) Image quality of vessel wall delineation must be adequate for clinical diagnosis of plaque. Both SNR and resolution challenges must be optimized for vessel wall boundary and plaque components depiction across multiple arteries.

In this study, we optimized 3D-MERGE, T2-weighted VISTA and SNAP into a 3D multi-contrast protocol combined with the advantage of a higher SNR and larger coverage neurovascular coil [18] to achieve a time efficient solution for joint intra- and extracranial VWI. The performance of this joint 3D plaque CMR protocol was investigated on stroke patients to test blood suppression effectiveness and vessel wall delineation ranging from the extracranial carotid to intracranial arteries.

\section{Methods \\ Study population}

In this Institutional Review Board approved study, 2 healthy volunteers (26 year-old males) were recruited to optimize sequence parameters for blood suppression, SNR, and wall delineation. Afterwards, 25 patients (17 males, age: $26-79$ years, median: 55 years) with recent (within 2 weeks) ischemic stroke or Transient Ischemic Attack (TIA) were consecutively recruited after informed consent.

\section{Sequence optimization}

The improved MSDE (iMSDE) [19] preparation in 3DMERGE (Fig. 1(a)) was implemented along all three orthogonal directions. A cumulative first order gradient moment $\left(\mathrm{M}_{1}\right)$ of $500 \mathrm{mT}^{* *} \mathrm{~ms}^{2} / \mathrm{m}, 1000 \mathrm{mT}^{*} \mathrm{~ms}^{2} / \mathrm{m}$ and $1500 \mathrm{mT}^{*} \mathrm{~ms}^{2} / \mathrm{m}$ were evaluated for vessel wall and lumen SNR measured in both intra- and extracranial segments during healthy volunteer scans, where 20 cross sectional slices were extracted with equal interval along both sides of the intra- and extracranial vasculatures. The preparation duration of iMSDE was minimized with the maximum gradient strength of $20 \mathrm{mT} / \mathrm{m}$ to reduce the signal loss caused by T2 decay or rephasing error induced by field inhomogeneity, eddy current and motion. After iMSDE preparation, a train of SPoiled GRadient echo (SPGR) with centric ordering was used for k-space data acquisition in 3D-MERGE [11].

For T2-weighted VISTA (Fig. 1(b)), FSD gradients were applied along the readout direction to efficiently suppress the excited blood spins within the imaging volume. After establishment of Static Pseudo Steady State (SPSS), the hard refocusing RF pulses were performed with Reduced Flip Angle (RFA) scheme. The refocusing flip angle and the echo train length were optimized using Extended Phase Graph (EPG) [20-23] in terms of SNR efficiency, which defined as a product of central echo intensity and a ratio between acquisition duration in each shot and the shot duration/repetition time. In this simulation, the repetition time was fixed to $2500 \mathrm{~ms}$ and the T1 and T2 relaxation times of $1114 \mathrm{~ms}$ and $55 \mathrm{~ms}$ respectively [24] were used as simulation parameters for vessel wall. The optimized RFA scheme was further compared with Variable Flip Angle (VFA) scheme in healthy volunteers with the identical effective echo time and repetition time to evaluate the quality for vessel wall delineation.

For SNAP (Fig. 1(c)), both Inversion Recovery (IR) and reference acquisition were acquired in linear ordering to guarantee the signal polarity requirement and minimize the background phase difference between IR and reference images [25]. A B1 insensitive nonselective adiabatic inversion pulse was applied and the inversion time (TI) was selected to meet the tissue signal polarity requirement and blood outflow condition [26]. In volunteer study, the nonselective inversion RF pulse was compared with its selective counterpart to investigate the suppression performance for blood inflow artifacts.

\section{CMR}

To support VWI across multiple vasculatures, receiving coil had to provide sufficient longitudinal coverage and SNR performance. In this work, a recently developed 36-channel neurovascular coil was employed with improved SNR performance at both intra- and extracranial segments in comparison to the commercialized 8-channel carotid/head coil and 16-channel neurovascular coil [18]. The head and carotid elements of this 36-channel coil were activated as MR signal receivers. With this large coverage coil configuration, a single 3D Cartesian slab (Fig. 2) can be prescribed to cover at least Common Carotid Arteries (CCA), Internal Carotid Arteries (ICA) and Middle Cerebral Arteries (MCA). This extended longitudinal FOV along Foot-Head (FH) direction can cover 


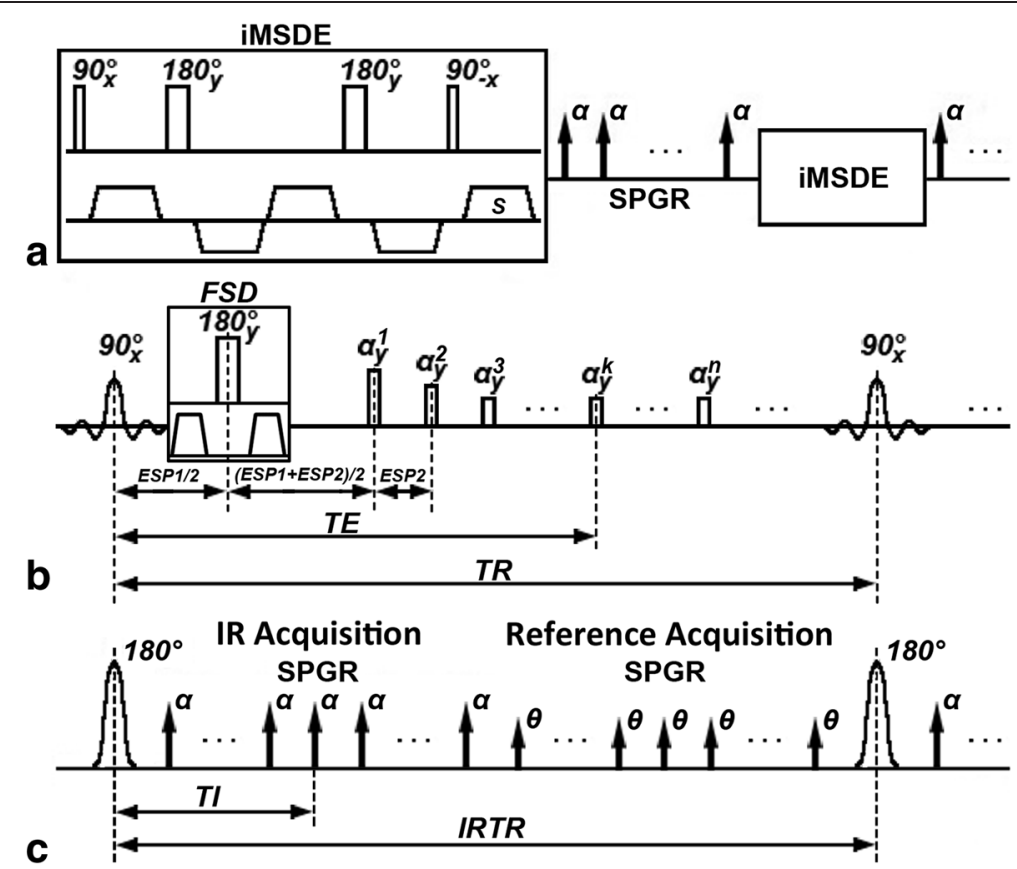

Fig. 1 Sequence diagram of 3D-MERGE, T2-weighted VISTA and SNAP. (a - c) corresponds respectively to the pulse sequence diagram of 3D-MERGE, T2-weighted VISTA and SNAP. The fat saturation scheme used both for 3D-MERGE and T2-weighted VISTA is spectral presaturation inversion recovery (SPIR) and for SNAP is water selective excitation in practice but they are not shown in this schematic figure

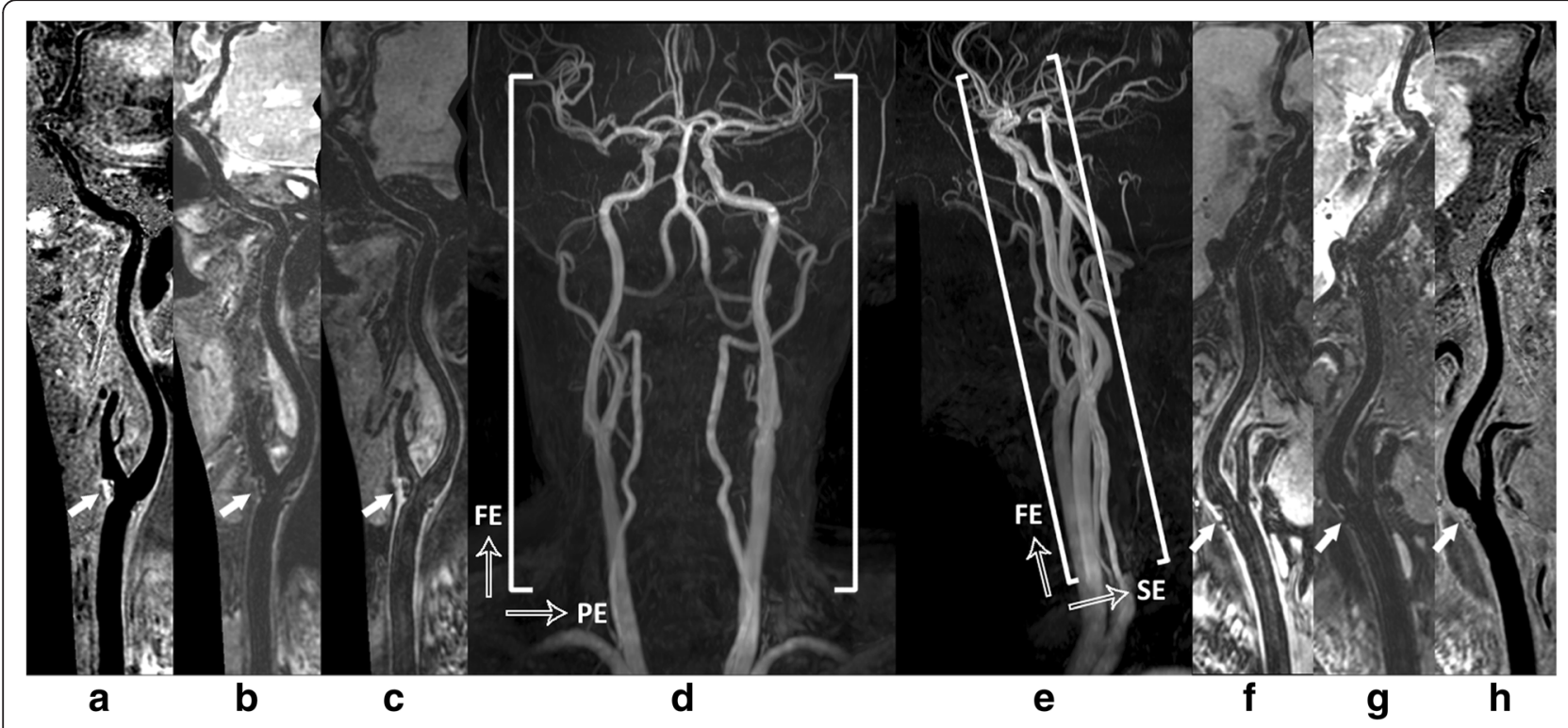

Fig. 2 Illustration of 3D multi-contrast imaging coverage. Three stations of 3D time-of-flight coronal maximum intensity projection (MIP) fusion (d) and sagittal MIP fusion (e) are illustrated here only for a clear definition of imaging FOV. The curved multi-planar reconstruction (MPR) examples show the right $(\mathbf{a}-\mathbf{c})$ and left $(\mathbf{f}-\mathbf{h})$ sides arteries ranging from CCA through ICA to MCA, where $(\mathbf{c}, \mathbf{f}),(\mathbf{b}, \mathbf{g})$, and $(\mathbf{a}, \mathbf{h})$ correspond to the results of 3D-MERGE, T2-weighted VISTA and SNAP respectively. Note the plaques were detected at bilateral carotid bifurcations on all different contrast weighted images as shown by solid arrows 
the most common sites for intra- and extracranial atherosclerosis [2] and avoid the potential imaging gaps or prolonged preparation time due to switching between carotid and head coils. Also, the overall scan time can be optimally controlled due to less number of phase encodes in Left-Right (LR) direction and thin slab thickness along Anterior-Posterior (AP) direction.

All experiments were performed on a $3 \mathrm{~T}$ MR scanner (Philips Achieva TX, Best, Netherland). All subjects were instructed to avoid swallowing during scans. Following 3 -plane survey, a fast large coverage 2D-PCA sequence was obtained for vessel localization (41 s). Then three coronal acquisitions of 3D-MERGE, T2-weighted VISTA and SNAP were performed with identical scan plane and same spatial resolution covering CCA, ICA and MCA. To maximize the available SNR and image quality, imaging acceleration methods were not considered in this study. The 3D multi-contrast protocol used herein (Table 1) can achieve isotropic $0.8 \mathrm{~mm}$ resolution to cover the major intracranial and entire carotid arteries within 15mins.

\section{Image analysis}

Both qualitative and quantitative analysis were performed at five locations, including CCA, carotid bifurcation (BULB), ICA C2, ICA C5 and MCA M1 segment [27, 28]. Each location was selected at the region with complex flow and various surrounding tissues, which is difficult for blood suppression and out wall boundary delineation. A curved MPR was firstly obtained on each 3D isotropic image dataset using open-source software Osirix [29]. Then $0.5 \mathrm{~mm}$ thick 2D cross-sectional slices were obtained at all five bilateral locations. The following qualitative and quantitative analysis were both conducted on these reconstructed 2D images.

Table 1 Imaging parameters for 3D multi-contrast sequences

\begin{tabular}{|c|c|c|c|}
\hline & 3D-MERGE & $\begin{array}{l}\text { T2-weighted } \\
\text { VISTA }\end{array}$ & SNAP \\
\hline$\overline{F O V}\left(F H x L R x A P m^{3}\right)$ & \multicolumn{3}{|c|}{$250 \times 160 \times 40$} \\
\hline $\begin{array}{l}\text { Resolution (FHxLRxAP } \mathrm{mm}^{3} \text { ) for } \\
\text { acquisition/reconstruction }\end{array}$ & \multicolumn{3}{|c|}{$0.8 \times 0.8 \times 0.8 / 0.4 \times 0.4 \times 0.4$} \\
\hline $\mathrm{TE}(\mathrm{ms})$ & 4.2 & $255 / 86^{a}$ & 4.5 \\
\hline TR (ms) & 9 & 2500 & $\begin{array}{c}10 / \\
1987^{b}\end{array}$ \\
\hline Echo spacing (ms) & NA & $10.8 / 3.6^{c}$ & NA \\
\hline Flip angle (deg) & 6 & 32 & $11 / 5^{d}$ \\
\hline Turbo factor & 90 & $130+4^{e}$ & 98 \\
\hline Averages & 2 & 1 & 2 \\
\hline Scan time (min) & $3: 10$ & $4: 32$ & $6: 47$ \\
\hline
\end{tabular}

${ }^{a}$ Effective echo time/Equivalent echo time

${ }^{b}$ SPGR repetition time/inversion recovery repetition time (IRTR)

'Echo spacing for the first/other refocusing interval

${ }^{d}$ Flip angle used in IR/reference acquisition

${ }^{\mathrm{e}}$ Number of echoes used in each shot for acquisition/SPSS start-up
Qualitative analysis was used to evaluate image quality on vessel wall delineation at each location for each sequence. All images were independently reviewed by two observers both with four-year experience in VWI. Image quality was scored using a four-level criteria. Score level from 4 to 1 corresponded to excellent, good, adequate and inadequate image quality. More specifically, excellent rated images showed clear vessel wall delineation for entire boundary. Good MR images represented good vessel wall delineation with only small part of obscure/invisible boundary. Adequate rated images stood for reasonable image quality on vessel wall visualization involving some part but less than a quadrant of obscure/invisible boundary. Finally, inadequate were acquisitions in which most of the vessel wall boundaries could not be seen.

Quantitative analysis was focused on evaluation of blood suppression effectiveness at each location. SNR measurements were performed with the free software ImageJ (version 1.48, National Institutes of Health, Bethesda, MD). Lumen signal $\left(\mathrm{S}_{\mathrm{l}}\right)$ was measured as the mean intensity within a Region-Of-Interest (ROI) manually drawn to cover the whole arterial lumen. For SNAP, $\mathrm{S}_{\mathrm{I}}$ measured on negative images were transformed into its opposite sign to avoid negative value. Arterial wall signal $\left(S_{w}\right)$ was measured as the mean signal intensity of pixels manually selected on the middle path of vessel wall boundary. The $\mathrm{SNR}$ of wall $\left(\mathrm{SNR}_{\mathrm{w}}\right)$ and lumen $\left(\mathrm{SNR}_{\mathrm{l}}\right)$ can be calculated as $\mathrm{SNR}_{\mathrm{w}}=\mathrm{S}_{\mathrm{w}} / \sigma_{\mathrm{n}}$ and $\mathrm{SNR}_{\mathrm{l}}=\mathrm{S}_{\mathrm{l}} / \sigma_{\mathrm{n}}$ respectively, where $\sigma_{\mathrm{n}}$ is the standard deviation of noise. Because of the inhomogeneous noise distribution due to spatially varying sensitivity map and post-processing image filter, $\sigma_{\mathrm{n}}$ was measured as the standard deviation within a ROI manually placed in the adjacent white matter for intracranial segment and muscle for extracranial carotid segment instead of the background air [30, 31]. The CNR between wall and lumen $\left(\mathrm{CNR}_{\mathrm{w}-1}\right)$ at each location were calculated as $\mathrm{CNR}_{\mathrm{w}-1}=\mathrm{SNR}_{\mathrm{w}}-\mathrm{SNR}_{\mathrm{l}}$ for 3D-MERGE and T2weighted VISTA while as $\mathrm{CNR}_{\mathrm{w}-\mathrm{l}}=\mathrm{SNR}_{\mathrm{w}}+\mathrm{SNR}_{\mathrm{l}}$ for SNAP due to opposite signal polarity of lumen and wall.

\section{Statistical analysis}

MATLAB (R2013b, Mathworks, Natick, MA) was used to perform the statistical analysis. $\mathrm{SNR}_{\mathrm{w}}$ and $\mathrm{SNR}_{\mathrm{l}}$ were compared at each location of one sequence using two-sided Wilcoxon signed rank test to evaluate the blood suppression performance. In all tests, statistical significance was defined at $\mathrm{P}<0.05$ level. Results are presented as column bar with error bar or mean \pm standard deviation at each location.

\section{Results}

\section{Sequence optimization}

The volunteer study indicated that the blood can be suppressed similarly but the Cerebral Spinal Fluid (CSF) can 


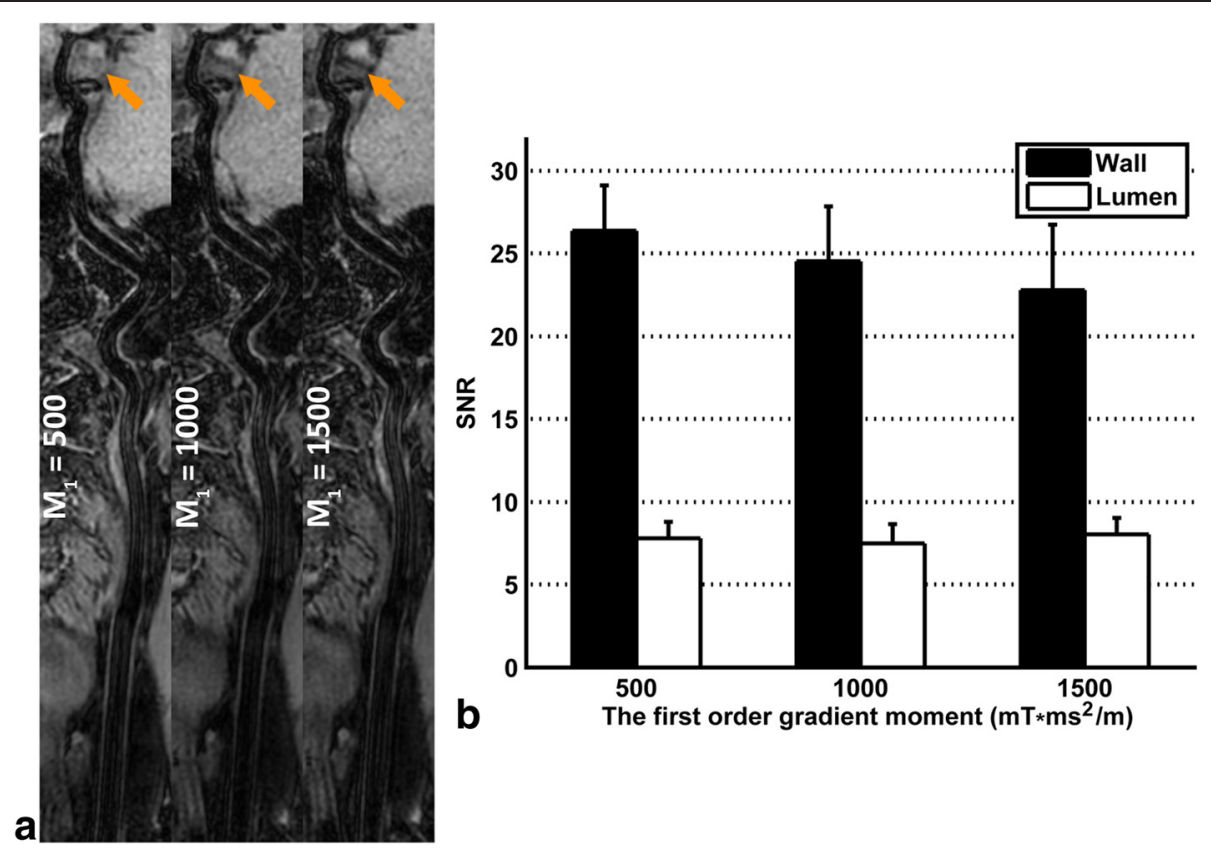

Fig. 3 Comparison of 3D-MERGE using different first order gradient moment $\left(M_{1}\right)$ values. A representative comparison for $M_{1}$ values ranging from $500 \mathrm{mT}^{*} \mathrm{~ms}^{2} / \mathrm{m}$ to $1500 \mathrm{mT}^{*} \mathrm{~ms}^{2} / \mathrm{m}$ are illustrated in (a). Note that CSF can be better suppressed when using larger $\mathrm{M}_{1}$ value (marked in yellow arrows). The vessel wall and lumen SNR are measured when different $M_{1}$ values are used and compared in (b)
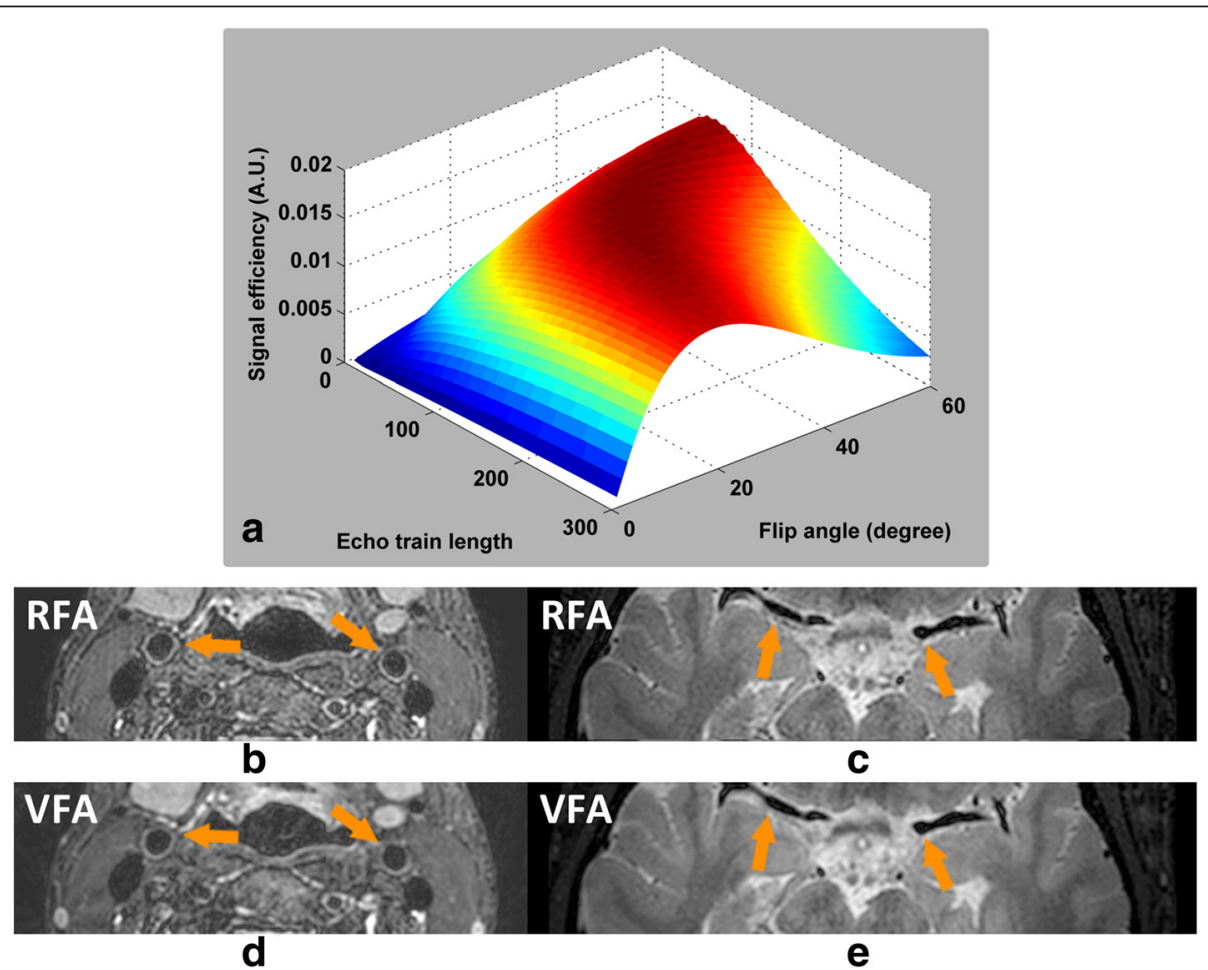

Fig. 4 Optimization of T2-weighted VISTA echo train. Panel (a) shows the optimization of RFA scheme on echo train length and refocusing flip angle. The optimized RFA scheme $(\mathbf{b}, \mathbf{c})$ can further improve the sharpness of vessel wall boundary (marked in yellow arrows) but cause SNR loss compared to VFA scheme (d, e) 


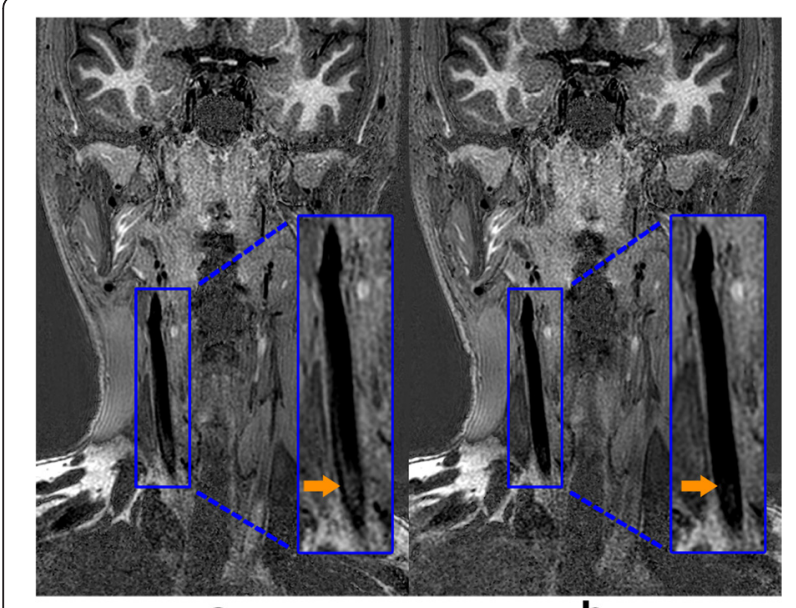

a

b

Fig. 5 Comparison of SNAP between selective and non-selective inversion pulse. The non-selective (b) inversion pulse can further improve the suppression of blood inflow artifacts in comparison to its selective counterpart (a)

be better suppressed with the $M_{1}$ value increasing, as shown in Fig. 3(a), which would improve the delineation of intracranial vessel wall. Also, the preparation duration of iMSDE was longer when using larger $M_{1}$ value so that the vessel wall SNR was penalized as shown in Fig. 3(b). With these considerations, the optimal $M_{1}$ value was selected as $1000 \mathrm{mT}^{* *} \mathrm{~ms}^{2} / \mathrm{m}$ in this study.
The EPG simulation showed that an inversely proportional relationship between refocusing flip angle and echo train length can provide a better tradeoff between the SNR of vessel wall and acquisition efficiency (Fig. 4(a)). Based on this result, a constant $32^{\circ}$ RFA scheme with echo train length of 130 were used in this study. Also, the smaller refocusing flip angle would be beneficial for flow suppression with this relatively long echo time [32, 33]. In addition, this optimized RFA scheme can help to improve the outer wall boundary definition in comparison to VFA scheme, particularly for the intracranial arteries surrounded with CSF (Fig. 4(b-e)), because the first half high frequency $\mathrm{k}$-space data acquired before the echo time was enhanced by RFA.

For SNAP optimization, the nonselective inversion pulse can better remove the blood inflow artifacts (Fig. 5) due to its extended inversion band and this would particularly benefit for SNAP imaging in this large coverage scenario comparing to the conventional selective inversion pulse.

\section{CMR}

The comparison result between 16- and 36-channel neurovascular coil using the previously optimized sequence parameters further demonstrated that the developed coil can outperform the commercialized one with improved SNR particularly at extracranial segments (Fig. 6).

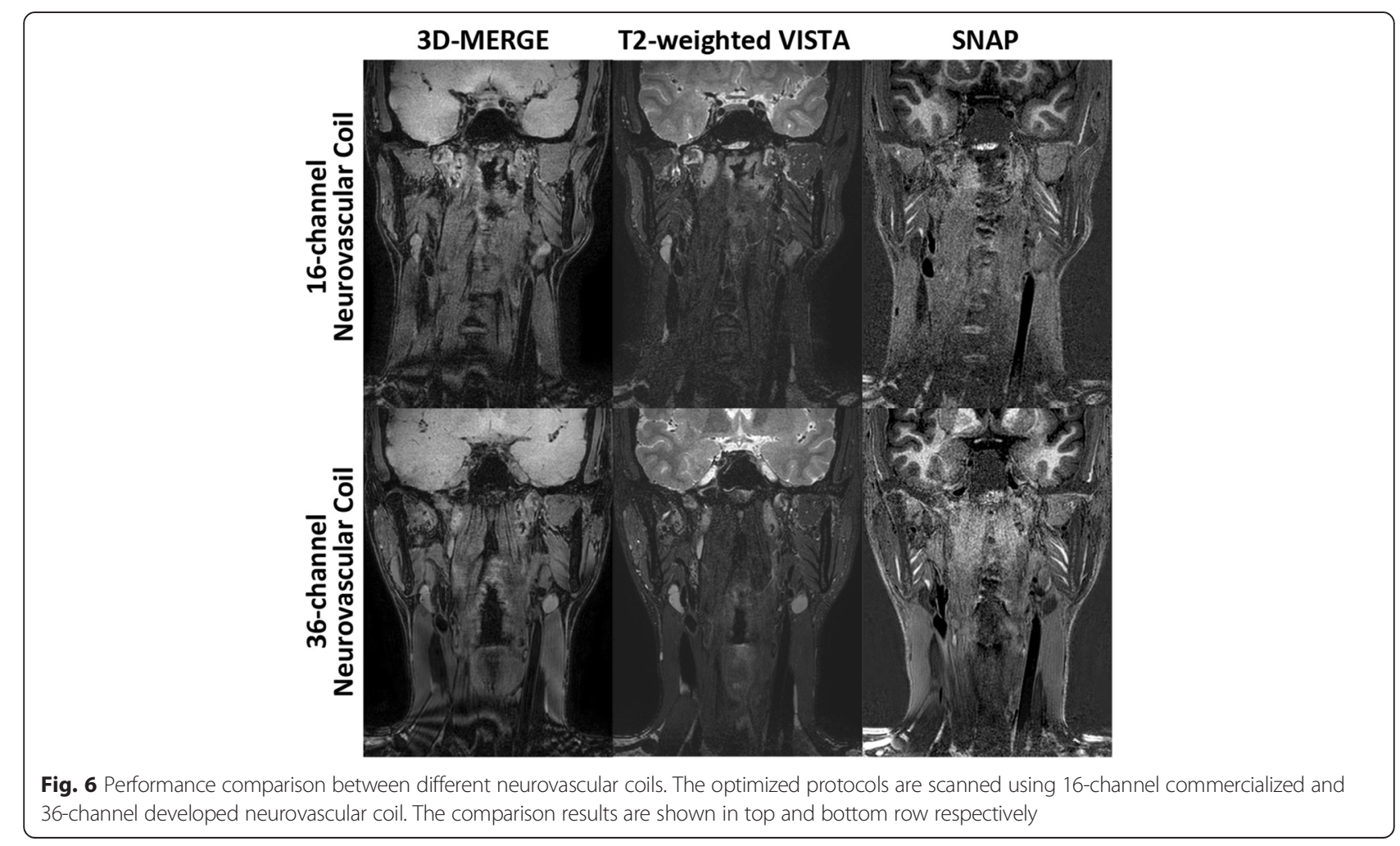


The 3D multi-contrast images were successfully acquired from all participants and all datasets were used for qualitative image scoring while two subjects were excluded from quantitative image analysis since SNR measurement cannot be performed at several locations due to occlusion. Good vessel wall delineation and blood suppression over the entire arteries were found on all multi-contrast images (Fig. 7). An overview of vessel wall across multiple vascular beds could be provided by $3 \mathrm{D}$ curved MPR of multi-contrast images which would be beneficial for plaque distribution analysis and risk assessment (Figs. 8 and 9).

\section{Image quality}

In general, 3D multi-contrast VWI was found to provide good image quality at extracranial segments and adequate image quality at intracranial segment respectively (Table 2). In two patients, the images at extracranial carotid segments were affected by motion artifacts, which adversely influenced with vessel wall delineation. In seven patients, the vessel wall depictions on SNAP images at intracranial segment were found to be unilaterally (6 out of 7 ) or

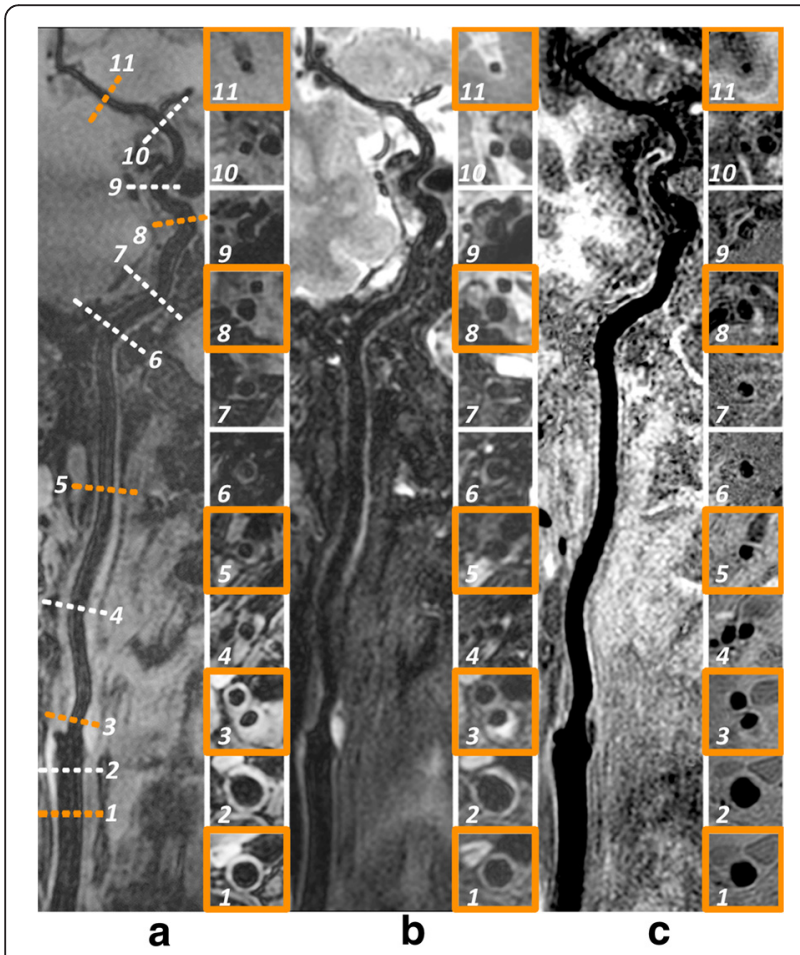

Fig. 7 Representative blood suppression and vessel wall delineation of the proposed 3D multi-contrast imaging. The curved MPR results in (a-c) show the overall blood suppression effect from 3D-MERGE, T2-weighted VISTA and SNAP sequence. The cross-sectional views at 11 locations were also provided with numbered subwindows in $(\mathbf{a}-\mathbf{c})$, including CCA, proximal and distal carotid bifurcation, ICA C1-C7 and MCA M1. The colored 5 locations were selected for quantitative analysis in this study. Note the plaque identified at location 3 illustrates different signal behaviors on different contrast weighted 3D sequences

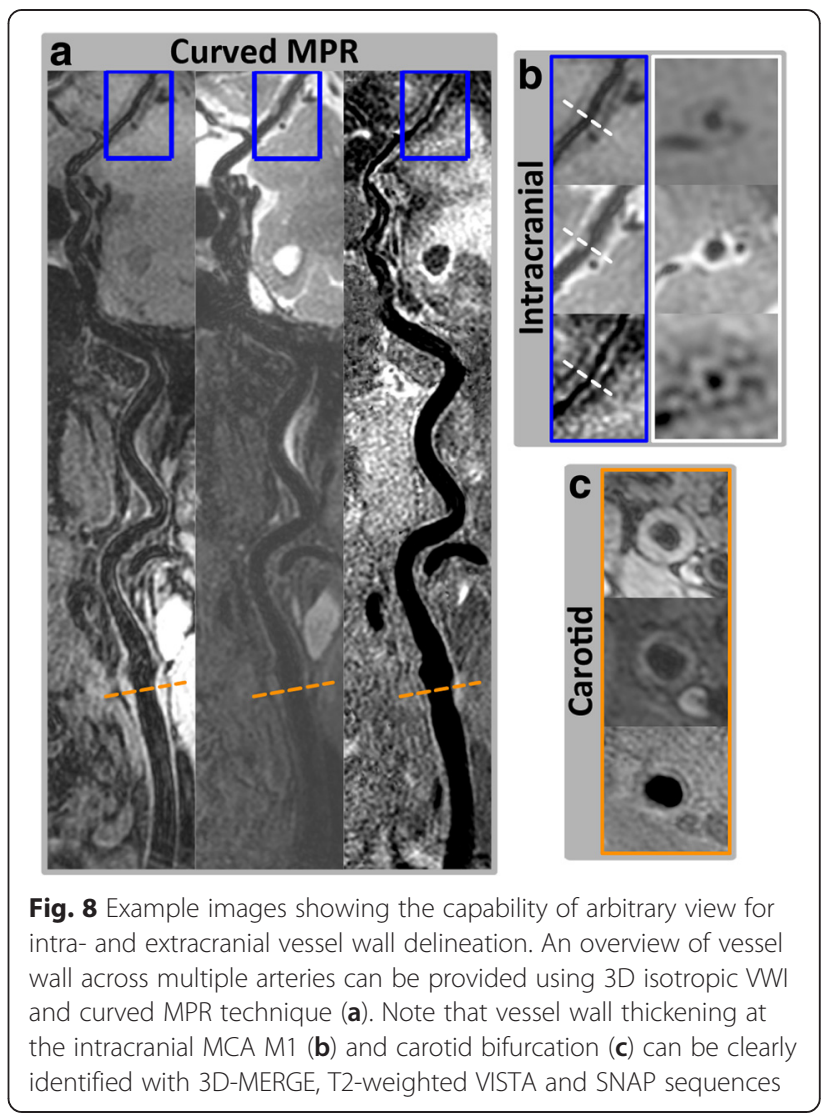

bilaterally (1 out of 7) inadequate. In other 18 patients, good or adequate images were successfully achieved in both intra- and extracranial segments for each sequence.

\section{Quantitative measurement}

The averaged $\mathrm{SNR}_{\mathrm{w}}$ from all five locations generally showed much greater value over the averaged $\mathrm{SNR}_{\mathrm{l}}$ (3D-MERGE: $\mathrm{SNR}_{\mathrm{w}}=19.07 \pm 6.13, \quad \mathrm{SNR}_{\mathrm{l}}=6.56 \pm 2.60, \quad \mathrm{P}<0.0001 ; \quad \mathrm{T} 2-$ weighted VISTA: $\mathrm{SNR}_{\mathrm{w}}=20.64 \pm 7.81, \mathrm{SNR}_{\mathrm{l}}=5.29 \pm 2.26$, $\mathrm{P}<0.0001 ; \quad$ SNAP: $\quad \mathrm{SNR}_{\mathrm{w}}=10.96 \pm 3.51, \quad \mathrm{SNR}_{\mathrm{l}}=17.79 \pm$ 9.44, $\mathrm{P}<0.0001$ ), indicating that blood suppression can be obtained for each $3 \mathrm{D}$ sequence. More specifically, $\mathrm{SNR}_{\mathrm{w}}$ and $\mathrm{SNR}_{1}$ measured at five locations on 3D-MERGE, T2weighted VISTA and SNAP images were shown in Fig. 10. The blood signal in all multi-contrast images can be suppressed effectively at all five measured locations and $\mathrm{CNR}_{\mathrm{w}-\mathrm{l}}$ calculated at these locations on each sequence were listed in Table 3.

\section{Discussion}

The 3D multi-contrast $\mathrm{BB}$ imaging technique can provide a rapid overview for joint intra- and extracranial VWI. The longitudinal coverage is $25 \mathrm{~cm}$, with isotropic spatial resolution of $0.8 \mathrm{~mm}$ and a total acquisition of 15 mins. This technique can potentially be used clinically to examine atherosclerotic plaques in both intra- and extracranial 


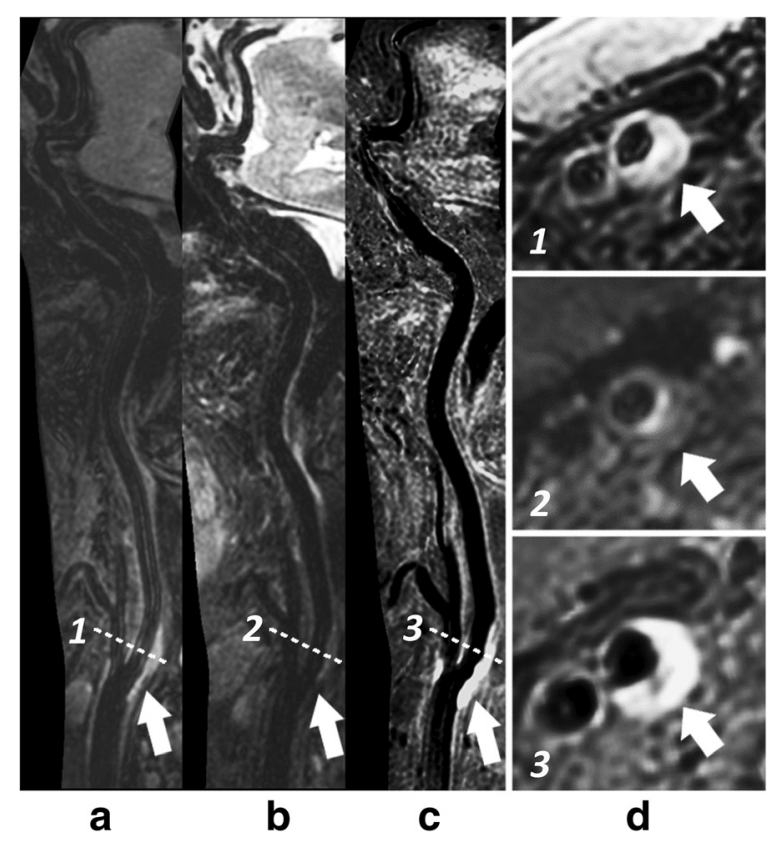

Fig. 9 Visualization of one local plaque in 3D-MERGE, T2-weighted VISTA and SNAP images. Both curved longitudinal and cross-sectional views of the intracranial and carotid arterial walls can be observed using MPR on 3D-MERGE (a), T2-weighted VISTA (b) and SNAP (c) images. The locations of the 3 cross-sectional images in (d) are marked by the dashed lines on the curved longitudinal images. IPH component and severe wall thickening distributed around the carotid bulb can be confirmed based on the 3D multi-contrast images

vascular beds for screening and/or diagnosis. Comparing to conventional angiographic techniques, this technique is able to provide information of the vessel wall and atherosclerotic plaque in addition to luminal stenosis. Direct visualization of vessel wall and the ability to assess high risk plaques may significantly improve the imaging ability to identify culprit lesions in subjects with stroke and/or

Table 2 The image quality score on vessel wall delineation for 3D multi-contrast sequences

\begin{tabular}{llcc}
\hline & 3D-MERGE & T2-weighted VISTA & SNAP \\
\hline Observer 1 & & & \\
CCA & $3.61 \pm 0.76$ & $3.39 \pm 0.85$ & $3.06 \pm 0.76$ \\
BULB & $3.03 \pm 0.56$ & $3.00 \pm 0.57$ & $2.67 \pm 0.59$ \\
ICA C2 & $2.92 \pm 0.49$ & $2.56 \pm 0.70$ & $2.78 \pm 0.49$ \\
ICA C5 & $3.36 \pm 0.61$ & $3.00 \pm 0.66$ & $2.86 \pm 0.51$ \\
MCA M1 & $2.44 \pm 0.38$ & $2.44 \pm 0.34$ & $2.33 \pm 0.42$ \\
Observer 2 & & & \\
CCA & $3.72 \pm 0.67$ & $3.42 \pm 0.90$ & $3.22 \pm 0.96$ \\
BULB & $3.72 \pm 0.57$ & $3.44 \pm 0.78$ & $3.22 \pm 0.83$ \\
ICA C2 & $3.14 \pm 1.08$ & $2.89 \pm 0.99$ & $3.50 \pm 0.71$ \\
ICA C5 & $3.42 \pm 0.86$ & $2.97 \pm 0.93$ & $3.08 \pm 0.94$ \\
MCA M1 & $2.64 \pm 0.95$ & $3.00 \pm 0.91$ & $2.86 \pm 0.89$ \\
\hline
\end{tabular}

TIA, and present clinicians with useful information to decide on the optimal treatment options. This technique may also be used in screening studies to identify subjects with high risk plaques.

The receiving coil plays an important role for joint intraand extracranial VWI both in its extended longitudinal coverage and improved SNR. The extended longitudinal coverage offers a rapid overview of bilateral arterial vessel walls with a single $3 \mathrm{D}$ slab acquisition and the improved SNR contributes to either smaller isotropic voxel size or accelerated imaging. The isotropic dataset can also be beneficial for improving the individual adaptability of wall visualization via curved MPR along vessels. To meet these two major concerns, a recently developed 36-channel coil is adopted in this study with a neurovascular mode to cover the entire intra- and extracranial arteries as well as its dedicated carotid coil elements to provide high SNR imaging.

The 3D multi-contrast information including T1, T2 and heavy $\mathrm{T} 1$ weighting has the potential to identify different plaque components similarly to $2 \mathrm{D}$ multi-contrast technique [14-16], but can cover more arteries from extracranial carotid to intracranial segments (Figs. 7, 8 and 9). As shown in Fig. 7, the plaque identified at carotid bifurcation with hyperintensity in T2-weighted VISTA and hypointensity in SNAP indicates loose matrix like component, while in Fig. 9 the hyperintense area in SNAP indicated by white arrow demonstrates the IPH component. Moreover, these 3D multi-contrast images offer complementary information on vessel wall delineation. The overall image quality after considering all three multi-contrast images is expected to be further improved compared to the separate qualitative score evaluated in this study.

Effective blood suppression and good or adequate image quality on vessel wall delineation can be achieved for all 3D multi-contrast sequences. Wall SNR is adequately higher over the lumen SNR at all five locations covering bifurcation and major torturous vessels from CCA to MCA for 3D-MERGE and T2-weighted VISTA. Lumen SNR measured in SNAP show larger values than wall SNR but actually they are in contrary polarity. This significant wall lumen difference/CNR reflects the inner wall boundary visibility. However, the wall lumen CNR for each sequence was not consistent across the five locations. The coil sensitivity profile can be another possible common explanation for the signal inhomogeneity of all sequences, especially for those segments from ICA C2 to ICA C5 which are much further away from the surface coil compared to other segments.

For 3D-MERGE, the lumen signal in ICA and MCA segments was found to be relatively larger than the values in CCA and carotid bifurcation segments $(\mathrm{P}<0.01)$. This is because iMSDE preparation module [19] depends on the dephasing of flowing blood spins within each luminal 


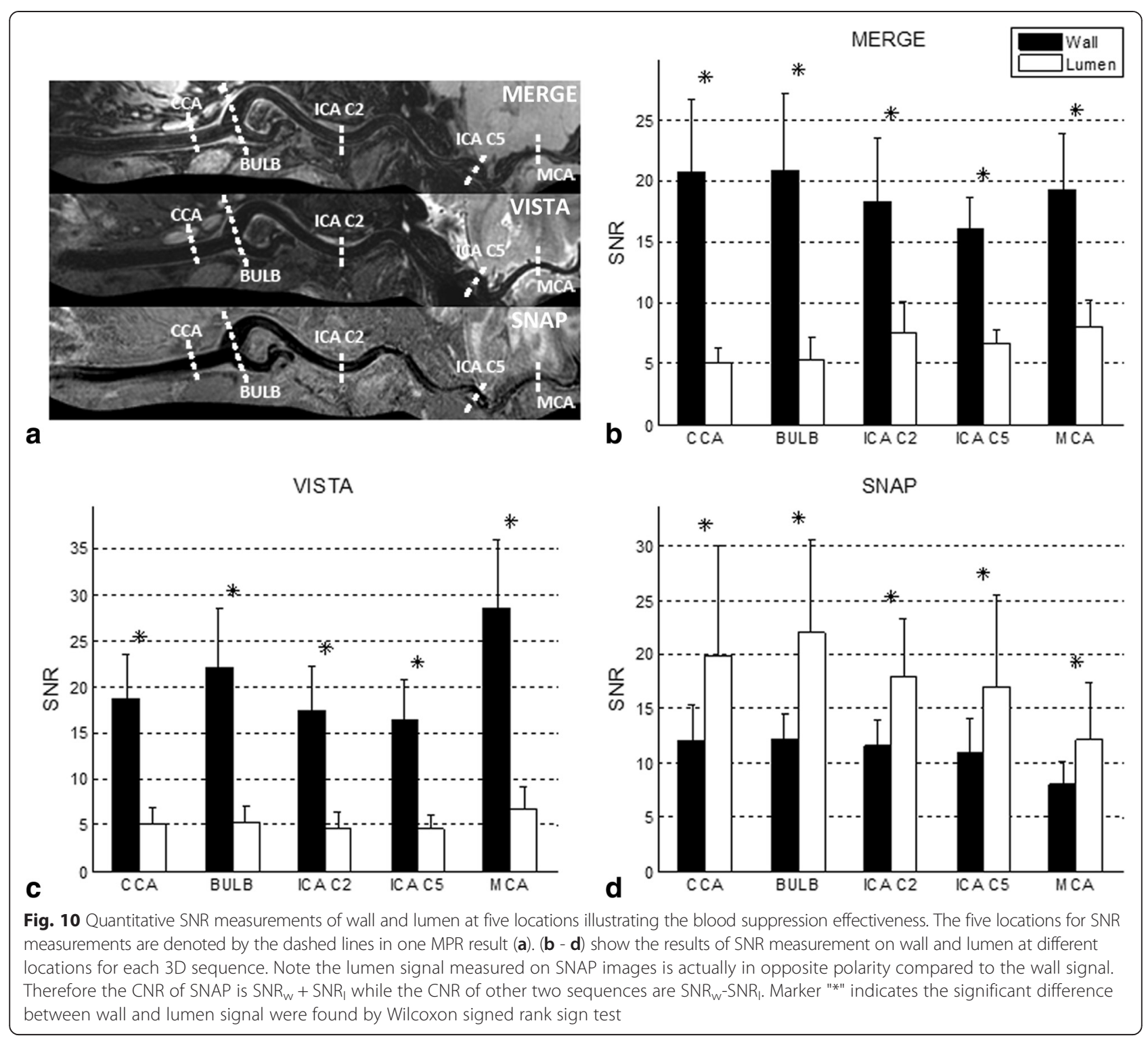

voxel, and the flow pattern in ICA and MCA tends to be more uniform than CCA and bifurcation. For T2-weighted VISTA, the high wall SNR measured in MCA segments $(\mathrm{P}<0.01)$ may be due to CSF contamination within the imaging voxel. VISTA performance in ICA C2, and ICA C5 was better than 3D-MERGE possibly because a spin echo based sequence is not as sensitive to susceptibility at

Table 3 The Wall-Lumen CNR for 3D multi-contrast sequences

\begin{tabular}{lccl}
\hline & 3D-MERGE & T2-weighted VISTA & \multicolumn{1}{c}{ SNAP } \\
\hline CCA & $15.56 \pm 5.20$ & $13.55 \pm 4.45$ & $31.86 \pm 12.40$ \\
BULB & $15.62 \pm 5.03$ & $16.81 \pm 5.14$ & $34.21 \pm 9.37$ \\
ICA C2 & $10.83 \pm 3.12$ & $12.80 \pm 3.56$ & $29.59 \pm 6.13$ \\
ICA C5 & $9.39 \pm 2.01$ & $11.72 \pm 3.46$ & $27.96 \pm 10.75$ \\
MCA M1 & $11.24 \pm 2.98$ & $21.86 \pm 6.15$ & $20.17 \pm 5.84$ \\
\hline
\end{tabular}

base of skull as a gradient based sequence. For SNAP, the lumen SNR in general showed a decaying trend along the blood flow direction which indicates the blood at distal part experience more than one IRTR cycle especially for large coverage scans leading to reduced blood suppression compared to proximal regions. On the other hand, in some cases, non-inverted blood can flow into CCA segments during the long TI time causing lumen signal polarity error at CCA. This gives rise to the fact that most of lumen signals remain in the positive value of corrected real images. Therefore, lumen SNR measured from negative value of corrected real images in CCA segments shows a smaller mean value and larger standard deviation in Fig. 10(d). This suggests that moving the patient table to ensure inflowing blood inversion before image acquisition can alleviate this problem. 
A limitation of large coverage is that the selected sequence parameters may not be optimal for vessel wall delineation in the entire intra- and extracranial vasculatures because the background tissue is different such as muscle in the neck compared to gray matter and white matter in the brain. Nevertheless, the sequence parameters used in this study are available for joint intra- and extracranial VWI with acceptable image quality. Another limitation is potential misregistration among these 3 multi-contrast images due to the sequential imaging scan order. However all three sequences were obtained with identical isotropic voxel dimensions that can allow registration by postprocessing. Spatial resolution used in this study might not be optimal for MCA imaging whose vessel caliber is smaller compared to extracranial vessels. Although higher spatial resolution can be achieved at the cost of increased scan duration, $0.8 \mathrm{~mm}$ isotropic scan was used in this study to meet the clinical requirement on scan duration while retaining image quality for the majority of the intra- and extracranial arteries. Several parallel imaging and/or compressed sensing methods to further enhance acquisition efficiency have been applied to applications including VWI $[34,35]$ or sequential multi-contrast imaging [36]. With these acceleration methods, the proposed 3D multicontrast VWI technique can be further improved regarding the voxel size, imaging FOV and scan duration. In addition, it is noteworthy to point out that further comparison with clinical gold-standard and/or histology is still required for validation of this technique for plaque components characterization.

Three investigated 3D imaging sequences can be used either individually or in combination depending on the specific clinical purpose. 3D-MERGE can provide clear vessel wall depiction in both intra- and extracranial vasculature that is beneficial for the plaque burden measurement. T2weighted VISTA and SNAP can be regarded as detection tools for high risk plaque components including necrotic core, IPH and calcification. However, it is not recommended to utilize T2-weighted VISTA alone due to the CSF contamination problem at intracranial segment. If contrast injection is permitted for patient scan, post-contrast T1 weighted imaging for example 3D-MERGE [37] can also be incorporated to identify lipid rich necrotic core and fibrous cap.

\section{Conclusions}

A 3D multi-contrast imaging technique at $3 \mathrm{~T}$ has been demonstrated for joint intra- and extracranial VWI with isotropic voxels, effective blood suppression, good or adequate image quality and clinically acceptable overall scan time. Its performance on vessel wall delineation and blood suppression has been demonstrated by qualitative image scoring and quantitative lumen/wall SNR measurements in this preliminary patient study. The proposed protocol has the potential to discriminate plaque components by multi-contrast analysis including T1, T2 and heavy T1 weighting. Further clinical verification is required for this promising tool on plaque distribution and plaque components characterization across multiple arteries.

\section{Competing interests}

The authors declare that they have no competing interests.

\section{Authors' contributions}

ZZ optimized MR pulse sequence, performed studies on healthy subjects, analyzed the data and drafted the manuscript. RL conceived the study and provided technical support in imaging study. XZ conceived the study and recruited patients for scan performance evaluation. LH performed studies on patients and helped with the image quality analysis. XW assisted the axial slice image preparation for qualitative and quantitative assessment. JW and NB provided technical support for sequence implementation. CY conceived the overall study and provided supervision and funding. All authors read and approved the final manuscript.

\section{Acknowledgements}

This work was supported by several grants including Beijing Municipal Science \& Technology Commission (No. Z131100005213001, No. D111107003111007), National Natural Science Foundation of China (No. 81271536) and NIH (No. HL103609 and No. NS072464). We thank Li Jiang, Shuo Chen, Zhensen Chen, Tingting Wu at Center for Biomedical Imaging Research (CBIR) and Dongxiang Xu at Vascular Imaging Lab (VIL) for their assistance on data handling. Authors also thank the constructive comments on statistics provided by Daniel S. Hippe at VIL.

\section{Author details}

${ }^{1}$ Center for Biomedical Imaging Research, Department of Biomedical Engineering, School of Medicine, Tsinghua University, Beijing, China. ${ }^{2}$ Department of Biomedical Engineering, Tsinghua University, Beijing, China. ${ }^{3}$ Department of Radiology, University of Washington, Seattle, WA, USA. ${ }^{4}$ Philips Research North America, Briarcliff Manor, NY, USA.

Received: 15 October 2014 Accepted: 1 May 2015

Published online: 27 May 2015

\section{References}

1. Arenillas JF. Intracranial atherosclerosis: current concepts. Stroke. 2011:42:S20-3.

2. Qureshi Al Caplan LR. Intracranial atherosclerosis. Lancet. 2014;383:984-98.

3. Man BL, Fu YP, Chan YY, Lam W, Hui AC, Leung WH, et al. Lesion patterns and stroke mechanisms in concurrent atherosclerosis of intracranial and extracranial vessels. Stroke. 2009;40:3211-5.

4. Bodle JD, Feldmann E, Swartz RH, Rumboldt Z, Brown T, Turan TN. High-resolution magnetic resonance imaging: an emerging tool for evaluating intracranial arterial disease. Stroke. 2013:44:287-92.

5. Ryu CW, Kwak HS, Jahng GH, Lee HN. High-resolution MRI of intracranial atherosclerotic disease. Neurointervention. 2014;9:9-20.

6. Yuan C, Mitsumori LM, Beach KW, Marvilla KR. Carotid atherosclerotic plaque: noninvasive MR characterization and identification of vulnerable lesions. Radiology. 2001;221:285-99.

7. Saam T, Hatsukami TS, Takaya N, Chu B, Underhill H, Kerwin WS, et al. The vulnerable, or high-risk, atherosclerotic plaque: noninvasive MR imaging for characterization and assessment. Radiology. 2007;244:64-77.

8. Kerwin WS, Hatsukami T, Yuan C, Zhao XQ. MRI of carotid atherosclerosis. AJR Am J Roentgenol. 2013;200:W304-13.

9. Qiao Y, Steinman DA, Qin Q, Etesami M, Schär M, Astor BC, et al. Intracrania arterial wall imaging using three-dimensional high isotropic resolution black blood MRI at 3.0 Tesla. J Magn Reson Imaging. 2011;34:22-30.

10. Qiao Y, Zeiler SR, Mirbagheri S, Leigh R, Urrutia V, Wityk R, et al. Intracranial plaque enhancement in patients with cerebrovascular events on highspatial-resolution MR images. Radiology. 2014:271:534-42.

11. Balu N, Yarnykh VL, Chu B, Wang J, Hatsukami T, Yuan C. Carotid plaque assessment using fast 3D isotropic resolution black-blood MRI. Magn Reson Med. 2011;65:627-37. 
12. Fan Z, Zhang Z, Chung YC, Weale P, Zuehlsdorff S, Carr J, et al. Carotid arterial wall MRI at 3 T using 3D variable-flip-angle turbo spin-echo (TSE) with flowsensitive dephasing (FSD). J Magn Reson Imaging. 2010;31:645-54

13. Wang J, Börnert P, Zhao H, Hippe DS, Zhao X, Balu N, et al. Simultaneous noncontrast angiography and intraplaque hemorrhage (SNAP) imaging for carotid atherosclerotic disease evaluation. Magn Reson Med. 2013;69:337-45.

14. Zhao X, Underhill HR, Zhao Q, Cai J, Li F, Oikawa M, et al. Discriminating carotid atherosclerotic lesion severity by luminal stenosis and plaque burden: a comparison utilizing high-resolution magnetic resonance imaging at 3.0 Tesla. Stroke. 2011;42:347-53.

15. Zhao X, Underhill HR, Yuan C, Oikawa M, Dong L, Ota H, et al. Minimization of MR contrast weightings for the comprehensive evaluation of carotid atherosclerotic disease. Invest Radiol. 2010;45:36-41.

16. Ota $\mathrm{H}$, Yarnykh VL, Ferguson MS, Underhill HR, Demarco JK, Zhu DC, et al. Carotid intraplaque hemorrhage imaging at 3.0-T MR imaging: comparison of the diagnostic performance of three T1-weighted sequences. Radiology. 2010;254:551-63.

17. Bornstedt A, Burgmaier M, Hombach V, Marx N, Rasche V. Dual stack black blood carotid artery CMR at $3 \mathrm{~T}$ : application to wall thickness visualization. J Cardiovasc Magn Reson. 2009;11:45-52.

18. Wang X, Li R, Hayes C, Balu N, Zhao X, Yuan C. A new designed 36-channel neurovascular coil at $3 \mathrm{~T}$. In Proceedings of the $20^{\text {th }}$ Annual Meeting of ISMRM 2012, abstract 2787

19. Wang J, Yarnykh VL, Yuan C. Enhanced image quality in black-blood MR using the improved Motion-Sensitized Driven-Equilibrium (iMSDE) sequence. J Magn Reson Imaging. 2010;31:1256-63.

20. Mihai G, Chung YC, Merchant A, Simonetti OP, Rajagopalan S. T1-weightedSPACE dark blood whole body magnetic resonance angiography (DB-WBMRA): initial experience. J Magn Reson Imaging. 2010;31:502-9.

21. Busse RF, Hariharan $H, V u A$, Brittain JH. Fast spin echo sequences with very long echo trains: design of variable refocusing flip angle schedules and generation of clinical T2 contrast. Magn Reson Med. 2006;55:1030-7

22. Hennig J, Weigel M, Scheffler K. Calculation of flip angles for echo trains with predefined amplitudes with the extended phase graph (EPG)algorithm: principles and applications to hyperecho and TRAPS sequences. Magn Reson Med. 2004;51:68-80.

23. Weigel M. Extended phase graphs: dephasing, RF pulses, and echoes pure and simple. J Magn Reson Imaging. 2015;41:266-95.

24. Kvernby S, Warntjes MJ, Haraldsson H, Carlhäll CJ, Engvall J, Ebbers T. Simultaneous three-dimensional myocardial T1 and T2 mapping in one breath hold with 3D-QALAS. J Cardiovasc Magn Reson. 2014;16:102.

25. Kellman P, Arai AE, McVeigh ER, Aletras AH. Phase-sensitive inversion recovery for detecting myocardial infarction using gadoliniumdelayed hyperenhancement. Magn Reson Med. 2002;47:372-83.

26. Wang J, Ferguson MS, Balu N, Yuan C, Hatsukami TS, Börnert P. Improved carotid intraplaque hemorrhage imaging using a slab-selective phasesensitive inversion-recovery (SPI) sequence. Magn Reson Med. 2010;64:1332-40.

27. Bouthillier A, van Loveren HR, Keller JT. Segments of the internal carotid artery: a new classification. Neurosurgery. 1996;38:425-32.

28. Huber P, Krayenbühl H, Yaşargil MG. Cerebral Angiography. 2nd edition. Thieme. 1982. p. 105-23.

29. Rosset A, Spadola L, Ratib O. OsiriX: an open-source software for navigating in multidimensional DICOM images. J Digit Imaging. 2004;17:205-16

30. Heverhagen JT. Noise measurement and estimation in MR imaging experiments. Radiology. 2007;245:638-9.

31. Dietrich O, Raya JG, Reeder SB, Reiser MF, Schoenberg SO. Measurement of signal-to-noise ratios in MR images: influence of multichannel coils, parallel imaging, and reconstruction filters. J Magn Reson Imaging. 2007;26:375-85

32. Storey P, Atanasova IP, Lim RP, Xu J, Kim D, Chen Q, et al. Tailoring the flow sensitivity of fast spin-echo sequences for noncontrast peripheral MR angiography. Magn Reson Med. 2010;64:1098-108.

33. Busse RF. Flow sensitivity of CPMG sequences with variable flip refocusing and implications for CSF signal uniformity in 3D-FSE imaging. In Proceedings of the $14^{\text {th }}$ Annual Meeting of ISMRM 2006, abstract 2430.

34. Makhijani MK, Balu N, Yamada K, Yuan C, Nayak KS. Accelerated 3D MERGE carotid imaging using compressed sensing with a hidden Markov tree model. J Magn Reson Imaging. 2012;36:1194-202.
35. Li B, Dong $L$, Chen B, Ji S, Cai W, Wang Y, et al. Turbo fast three-dimensional carotid artery black-blood MRI by combining three-dimensional MERGE sequence with compressed sensing. Magn Reson Med. 2013;70:1347-52.

36. Gong E, Huang F, Ying K, Wu W, Wang S, Yuan C. PROMISE: parallel-imaging and compressed-sensing reconstruction of multicontrast imaging using SharablE information. Magn Reson Med. 2015:73:523-35.

37. Yuan C, Wang J, Balu N. High Field Atherosclerotic Plaque MRI. Neuroimaging Clin N Am. 2012:22:271-84.

\section{Submit your next manuscript to BioMed Central and take full advantage of:}

- Convenient online submission

- Thorough peer review

- No space constraints or color figure charges

- Immediate publication on acceptance

- Inclusion in PubMed, CAS, Scopus and Google Scholar

- Research which is freely available for redistribution 